\title{
BMJ Open The CEA Second-Look Trial: \\ a randomised controlled trial of \\ carcinoembryonic antigen prompted reoperation for recurrent colorectal
}

\section{cancer}

\section{Tom Treasure, ${ }^{1}$ Kathryn Monson, ${ }^{2}$ Francesca Fiorentino, ${ }^{3}$ Christopher Russell ${ }^{4}$}

To cite: Treasure $\mathrm{T}$,

Monson K, Fiorentino F, et al. The CEA Second-Look Trial: a randomised controlled trial of carcinoembryonic antigen prompted reoperation for recurrent colorectal cancer. BMJ Open 2014;4:e004385. doi:10.1136/bmjopen-2013004385

- Prepublication history and additional material is available. To view please visit the journal (http://dx.doi.org/ 10.1136/bmjopen-2013004385)

The following paper published in the $B M J$ relates to this article: Treasure $\mathrm{T}$, Monson K, Fiorentino F, et al. Operating to remove recurrent colorectal cancer: have we got it right? BMJ 2014;348: g2085.

Received 1 November 2013 Revised 8 March 2014 Accepted 14 April 2014

CrossMark

For numbered affiliations see end of article.

Correspondence to Dr Tom Treasure; tom.treasure@gmail.com

\section{ABSTRACT}

Objective: In patients who have undergone a potentially curative resection of colorectal cancer, does a 'second-look' operation to resect recurrence, prompted by monthly monitoring of carcinoembryonic antigen, confer a survival benefit?

Design: A randomised controlled trial recruiting patients from 1982 to 1993 was recovered under the Restoring Invisible and Abandoned Trials (RIAT) initiative.

Setting: 58 hospitals in the UK.

Participants: From 1982 to 1993, 1447 patients were enrolled. Of these 216 met the criteria for carcinoembryonic antigen (CEA) elevation and were randomised to 'Aggressive' or 'Conventional' arms.

Interventions: 'Second-look' surgery with intention to remove any recurrence discovered.

Primary outcome measure: Survival.

Results: By February 1993, 91/108 patients had died in the 'Aggressive arm' and 88/108 in the

'Conventional' arm (relative risk=1.16, $95 \% \mathrm{Cl} 0.87$ to 1.37). By 2011 a further 25 randomised patients had died. Kaplan-Meier analysis showed no difference in long-term survival.

Conclusions: The trial was closed in 1993 following a recommendation from the Data Monitoring Committee that it was highly unlikely that any survival advantage would be demonstrated for CEA prompted second-look surgery. This conclusion was confirmed by repeat analysis of survival times after 20 years.

Trial registration number: ISRCTN76694943.

\section{INTRODUCTION}

The Working Party of the carcinoembryonic antigen (CEA) Second-Look Trial set the scene for their trial in their protocol in 1982 (Slack W, Bagshawe K, Baum M, et al. Protocol: a multicentre trial to evaluate the use of serial carcinoembryonic antigen assay as the prime indicator for second-look

\section{Strengths and limitations of this study}

- The carcinoembryonic antigen (CEA) SecondLook Trial was a well-planned and carefully executed study with a clear question and a welldefined outcome of interest.

- Second-look surgery prompted by the best available indicator of recurrence at the time conferred no survival advantage.

- A further strength, and a reason to publish this trial now, is that it shows that randomised trials in surgery can be carried out and that the result may be contrary to the beliefs and expectations of practitioners based on their uncontrolled observations.

- A limitation is that present day means of noninvasive detection of asymptomatic recurrence were not available at the time of the CEA Second-Look Trial. A recently reported randomised controlled trial (FACS) in which regular CEA and/or CT monitoring were compared with minimum follow-up showed no survival advantage associated with earlier detection through monitoring.

surgery in recurrent colorectal cancer (Trial Archive), 1982, unpublished work). The principal finding, that CEA monitoring to detect asymptomatic recurrence was not associated with improved survival, was announced in a letter to the Journal of the American Medical Association in 1994 by Northover, the then chief investigator. ${ }^{1}$ The writing of the trial for publication lapsed. We here report the trial under the Restoring Invisible and Abandoned Trials (RIAT) initiative. $^{23}$

It had been observed during the 1970s that the outlook for patients with colorectal cancer was not good. Only one in four patients survived for 5 years after diagnosis and radical surgery was observed to be 
Figure 2 Illustration of operative findings in six successive operations seeking recurrence of colorectal cancer. ${ }^{14}$
WANGENSTEEN ET AL.: SECOND LOOK PROCEDURE FOR CANCER

259

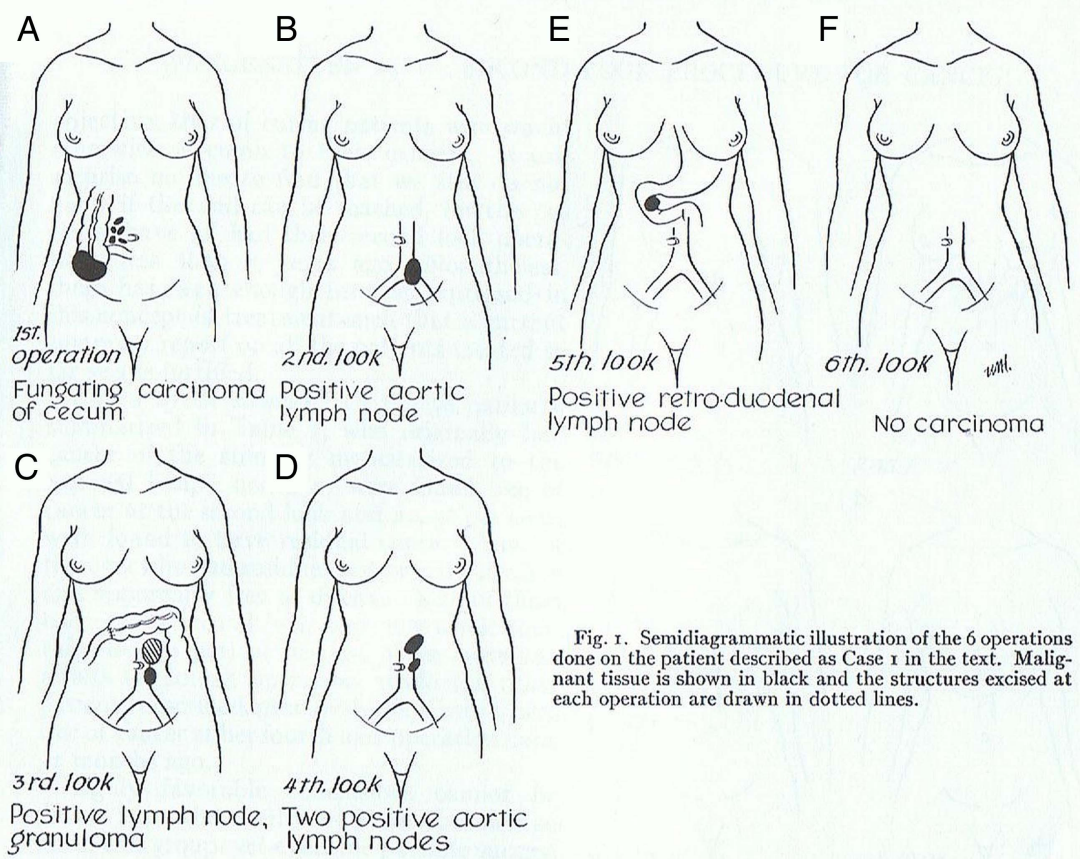

look laparotomy prompted by clinical indications. Others had not found CEA to be useful in this postoperative monitoring role. Even if efficacy of CEA-detected recurrence was accepted, there was still the unresolved question of effectiveness: if more patients were detected and there were more instances of resectable recurrence, did that lead to better survival and patient benefit? The conflicting interpretations of observational data resulted in calls for trials ${ }^{15} 2122$ including one within a $1981 \mathrm{NIH}$ Consensus Statement. ${ }^{20}$

The objective of the CEA Second-Look Surgery Trial was to determine whether, following potentially curative primary surgery for colorectal cancer, mortality could be decreased by a policy of second-look surgery prompted by rising serum CEA. The trial ran from 1982 to 1993. The main result, that there was no survival advantage, was reported in 1994 to the British Oncological Association $^{23}$ and was published in a letter to the Journal of the American Medical Association. ${ }^{1}$

Detection and reoperation for asymptomatic colorectal cancer recurrence has since become routine in the form of hepatic resection ${ }^{24}$ and pulmonary metastasectomy $^{25}$ but without evidence from controlled trials for either practice. ${ }^{2627}$ When doubts were raised about the security of the evidence in the $B M J$ in $2007^{26}$ a general belief existed that randomised controlled trials of the effectiveness of resection of liver or lung metastases were not possible and were not needed. These paired beliefs are brought into question by the previously unpublished CEA Second-Look Trial: a randomised trial had been carried out and the presumed benefit of surgery for cancer recurrence was not seen. ${ }^{123}$
Closure of the trial in 1993 and gaining access to the data in 2011

The RIAT restorative authors had been involved in various studies related to surgery for disseminated colorectal cancer $^{262829}$ including a conundrum as to whether discovery of an elevated CEA assay should prompt, or be considered a contraindication to, pulmonary metastasectomy. ${ }^{30}$ We knew the CEA trial had been recruiting in the 1980s but when we searched the literature for the result of the trial we found nothing later than $19944^{1{ }^{23}}$ In 2009 we contacted the chief investigator of the trial at the time of its closure (JMAN) and the present director of the University College London Cancer Trials Centre (JAL). We were informed that the data were irretrievably lost. However, $\mathrm{KM}$ was aware that CEA trial data were still in the department and after further enquiries RCGR gained access to anonymised electronic data in 2011. The process of data would be published as part of 'RIAT'. 23

Among the documents made available to the RIAT restorative authors were listed the members of the trial development group in the 1982 protocol (Slack et al, 1982, unpublished work) and the contributors to the 1994 manuscript (Northover J, Houghton J. Post operative CEA monitoring and second-look surgery in colorectal cancer: the effect on survival measured using a multicentre randomised trial (Manuscript), 6-7-1994, unpublished work). None of these individuals expressed an interest in resuming work on the trial or were in a position to do so. When we contacted them later to share the restored data with them no one raised any objection but on the contrary encouraged us to publish their findings. restoration is described later. It was agreed that the trial 


\section{METHODS}

\section{Trial intent and design}

The recruitment intentions and the trial protocol as presented here are essentially as written in the manuscript prepared in 1994 with the full intention of publishing the trial (Northover and Houghton, 1994, unpublished work). The text has been edited by the RIAT authors but no new material has been introduced.

The CEA Second-Look Trial was intended to recruit at least 2000 patients over 3 years and to follow them for 5 years. The study was specifically designed with late randomisation in order to maximise statistical power. It was originally intended to recruit 2000 patients with the anticipation that about $25 \%$ would show a CEA rise as the first evidence of possible recurrence. This number would have provided $95 \%$ power to detect an improvement in 2-year survival from the second-look procedure from $25 \%$ to $55 \%$ at $\alpha=0.05$. The protocol stated that for the trial to be stopped prematurely very stringent levels of significance $(p<0.001)$ would be used. Analyses of the randomised groups were to be by Kaplan-Meier lifetables and the logrank test on 'intention to treat' (Northover and Houghton, 1994, unpublished work).

Their intentions were explicitly set out as follows in 1981 (Slack WW, Public Health Service Grant Application to NIH, 1981, personal communication):

So far as society in general is concerned, if CEA monitoring is shown to be of benefit in this study, then it will be a powerful incentive to the great majority of surgeons who see no obvious advantage in routine CEA monitoring to adopt the technique; as colorectal cancer is the second commonest killing cancer in the Western world, the benefits would thus be enormous. If, however, CEA monitoring is shown to be of no long term therapeutic value then it should cease to be used in its presently available form, and patients will thereby be spared the "needless anxiety'"22 of premature knowledge of their impending death.

The CEA trial design (figure 3) was devised so that clinical follow-up would remain unbiased, and allow specific evaluation of the role of CEA-indicated surgery in the treatment of recurrent colorectal cancer. After potentially curative surgery for colorectal cancer, all eligible patients were to be monitored identically using conventional clinical follow-up together with regular CEA assay, performed centrally. Clinicians would not be informed of the result. When a 'significant' CEA rise was recorded, patients were to be randomised by the Trials Centre into either 'Aggressive' or 'Conventional' arms. In the case of patients in the 'Aggressive' arm, the clinician would immediately be informed of the CEA rise so that the patient could be urgently screened to exclude widespread metastatic disease or a non-malignant cause for the CEA rise. If neither was found, and the patient was medically fit for operation, the protocol required second-look surgery to locate and remove any treatable recurrence. In the case of patients in the 'Conventional' arm, the clinician would not be informed of the 'significant' CEA rise nor of the fact that they had been randomised to not have the CEA rise revealed.

The primary outcome was survival based on death certification through the Office of Population Censuses and Surveys (OPCS; now called the Office for National Statistics (ONS)). No subset analyses were planned.

\section{The intention as stated in the protocol was that the trial would produce}

A. A definitive answer concerning the effectiveness of CEA-prompted second-look surgery to improve survival.

B. An accurate picture of the 'lead time' produced by CEA compared to clinically indicated second-look surgery.

C. Further data relating CEA levels to tumour histology and topography.

D. A large database on the natural history of colorectal cancer (Slack et al, 1982, unpublished work).

The RIAT restorative authors regard (A) and (B) as planned analyses. The (C) and (D) statements give no indication as to the precise nature of analyses that might follow and are regarded as opportunities for explanatory subset analyses which were not in the event carried out.

\section{The conduct of the trial 1982-1993}

The trial was coordinated (initially) from the Cancer Research Campaign (CRC) Clinical Trials Centre at King's College Hospital. CEA assays were performed using a radioimmunoassay technique at a single centre at Charing Cross Hospital.

\section{Selection of patients}

All patients up to the age of 76 who had undergone a potentially curative resection for adenocarcinoma of the colon or rectum and who were fit and willing to adhere to the postoperative monitoring routine were eligible for the study. Patients were excluded if there was evidence of incurable distant spread, either preoperatively or during the primary operation, or if the CEA level failed to return to the normal range $(<10 \mathrm{ng} / \mathrm{mL})$ within 6 weeks of primary surgery. Patients who had previously received treatment for other types of cancer, apart from basal or squamous cell carcinoma of the skin or in situ carcinoma of the cervix adequately cone biopsied, were excluded from the study.

\section{Management of the primary tumour}

A preoperative blood sample for CEA assay was taken from all patients with suspected colorectal adenocarcinoma who otherwise fulfilled the trial entry criteria. This was a pragmatically designed study so surgeons were at liberty to use their normal operative technique and to employ preoperative or postoperative radiotherapy or adjuvant chemotherapy as was seen fit, however they were asked to remain consistent regarding the treatment used for any particular type of disease. If at laparotomy, a potentially curative resection was performed and 
subsequent histology confirmed the diagnosis of adenocarcinoma, the patient was given a full explanation of the study and could be registered.

\section{Consent}

The 1982 protocol includes a consent form (Consent form A) to be completed at registration and a further form (Consent form B) for patients who were randomised to a 'Second-Look Laparotomy'. There was a protocol amendment in which the word 'cancer' is to be replaced throughout by 'a growth' (Slack et al, 1982, unpublished work).

\section{Baseline data}

The surgeons carried out investigations to detect the presence of synchronous colorectal tumours (benign and malignant) and to exclude occult liver spread; (usually barium enema examination and ultrasound or CT of the liver). In addition, factors that could give raised CEA levels in the absence of recurrent colorectal cancer, such as chronic lung disease, cirrhosis, chronic pancreatitis and chronic renal failure were excluded by clinical questioning, chest X-ray, liver function tests, blood urea and electrolytes. Smoking habits and alcohol consumption were also recorded as heavy smoking or drinking, or a change in these habits, can influence CEA levels.

\section{Monitoring of patients}

Clinical follow-up of all patients continued in an identical manner (3 monthly for the first 2 years and 6 monthly for the next 3 years) while blood for CEA assay was drawn monthly for the first 3 years and 3 monthly for the next 2 years. If the patient remained well and the CEA was within normal limits as defined by a pretested algorithm, monitoring continued according to the schedule.

\section{CEA assay}

Ten millilitres of whole blood were taken from each patient. The serum was separated and sent to the Trials Centre in special plastic phials. After logging receipt, the samples were forwarded to the Medical Oncology Department at Charing Cross Hospital for assay. The results were returned to the Trials Centre for recording and action if appropriate. This centralised system ensured that all participating clinicians were kept blind to the CEA results for their patients. It also ensured quality control of the CEA assay as there was no possibility of interlaboratory variation.

Serum CEA values were measured by double antibody radioimmunoassay. ${ }^{31-33} \mathrm{~A}$ bank of serum samples has been retained at $-20^{\circ} \mathrm{C}$.

\section{Monitoring assay compliance prerandomisation}

Throughout the trial, compliance with blood sampling was monitored by the secretariat. Clinicians were reminded each month of the patients for whom samples were due; those who had missed the previous visit were highlighted as urgent. The percentage compliance for each participating patient was calculated as the number of samples received divided by those expected $\times 100$. The median time between samples was also calculated. Failure to achieve $50 \%$ of the expected samples was defined as poor compliance. Since the sensitivity to detect CEA rise in such patients was greatly reduced they were excluded from randomisation.

\section{'Significant' rises in CEA}

A rise in CEA was defined as 'significant' when the CEA level was greater than $10 \mathrm{ng} / \mathrm{mL}$ on two successive occasions and one of the following conditions was also met: the CEA level was greater than $20 \mathrm{ng} / \mathrm{mL}$ on each of two successive occasions or the level was rising and the highest value was more than $7 \mathrm{ng} / \mathrm{mL}$ above the lowest value ever recorded. If a 'significant' rise in CEA occurred, the record of the patient was reviewed at the Trials Centre and provided no evidence of suspected colorectal or other disease was recorded in the CRF, the patient was randomised either into an 'Aggressive' or 'Conventional' arm (figure 4).

\section{Randomisation}

Patients were randomised equally between the two arms (1:1). Patients whose compliance was between $50 \%$ and $70 \%$ or whose immediate postoperative sample had not been received within the 4-6-week guideline were randomised in a separate stratum. Randomisation was also stratified by participating clinician. A block size of two was used in order to maintain as close a balance as possible between the two treatment arms.

If the patient was randomised to the 'Aggressive' arm the clinician was informed of the rise immediately by telephone from the trial centre and subsequently in writing and was requested to contact the patient urgently. Patients were informed of their situation including the fact that they had been randomised within the trial to undergo a second-look procedure. This was then undertaken if the patient gave written informed consent. The surgeon carried out a full clinical workup to exclude the possibility of a non-malignant cause for the CEA rise (eg, change in smoking or drinking habit) and to identify any incurable distant spread. In the absence of these conditions the surgeon undertook a mini-laparotomy, proceeding to full laparotomy with macroscopic clearance of disease, should this be possible.

For patients randomised to the 'Conventional' arm no further action was taken; the clinician was neither informed that the CEA had risen nor that the patient had been randomised.

If at any stage a patient in the study developed clinical evidence of recurrent disease the clinician was at liberty to manage the patient according to usual practice. If the 


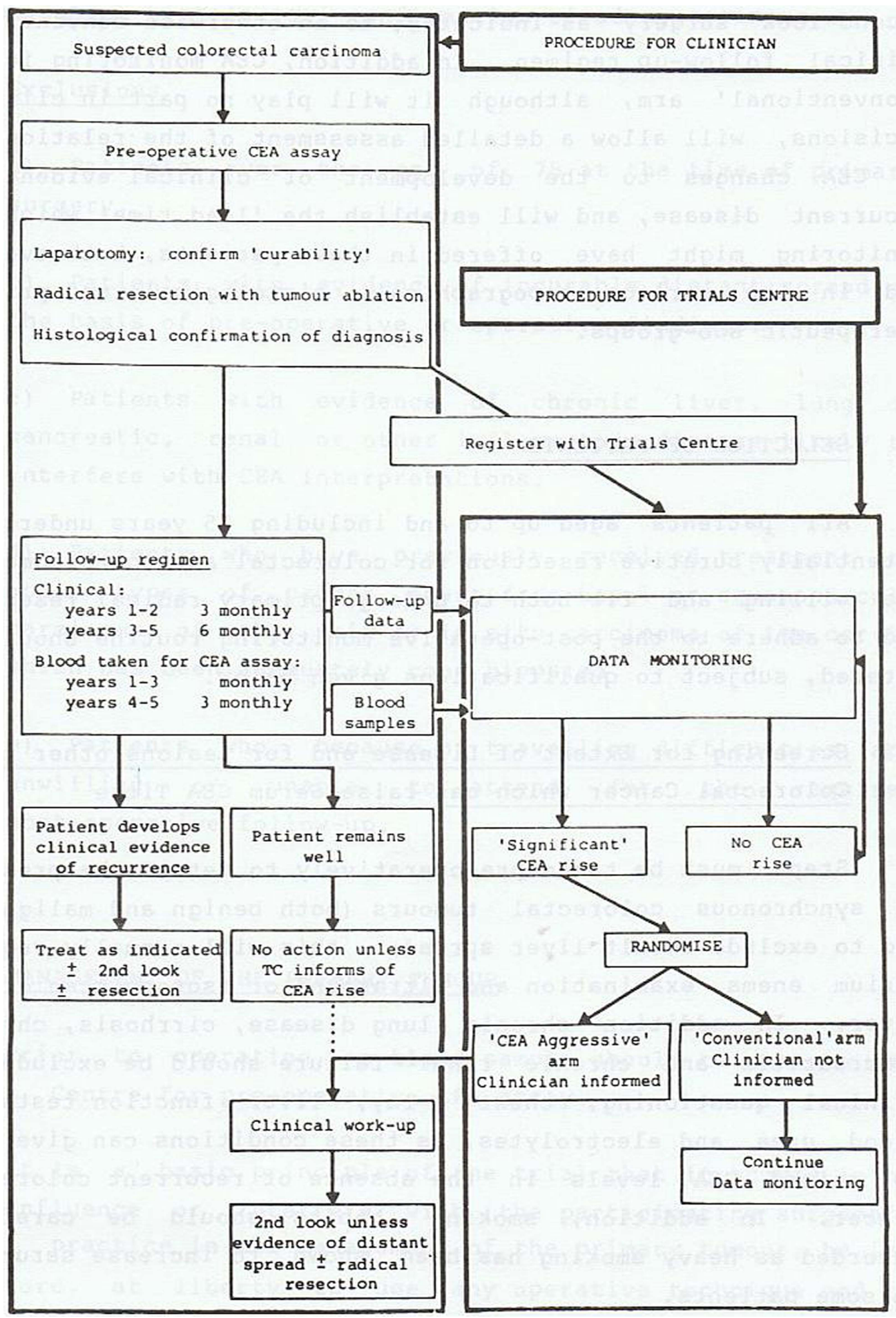

Figure 3 Flow diagram of the Second-Look Surgery Trial from the 1982 protocol (Slack et al, 1982, unpublished work).

disease was in the abdomen and was thought to be treatable by a second-look operation with re-resection, this was acceptable. By the nature of the trial design, the clinician was blind as to whether such patients had been randomised to the 'Conventional' arm of the trial or had not been randomised because the CEA had failed to denote the presence of recurrent disease.

\section{Second-look laparotomy}

The surgeon was expected to perform a thorough inspection of the abdominal cavity to locate any recurrent disease. Initially a mini-laparotomy was performed; if widespread tumour was detected all that was required prior to closure, was biopsy. Otherwise following a full excision, bimanual palpation of the old scar, inspection and palpation of the 


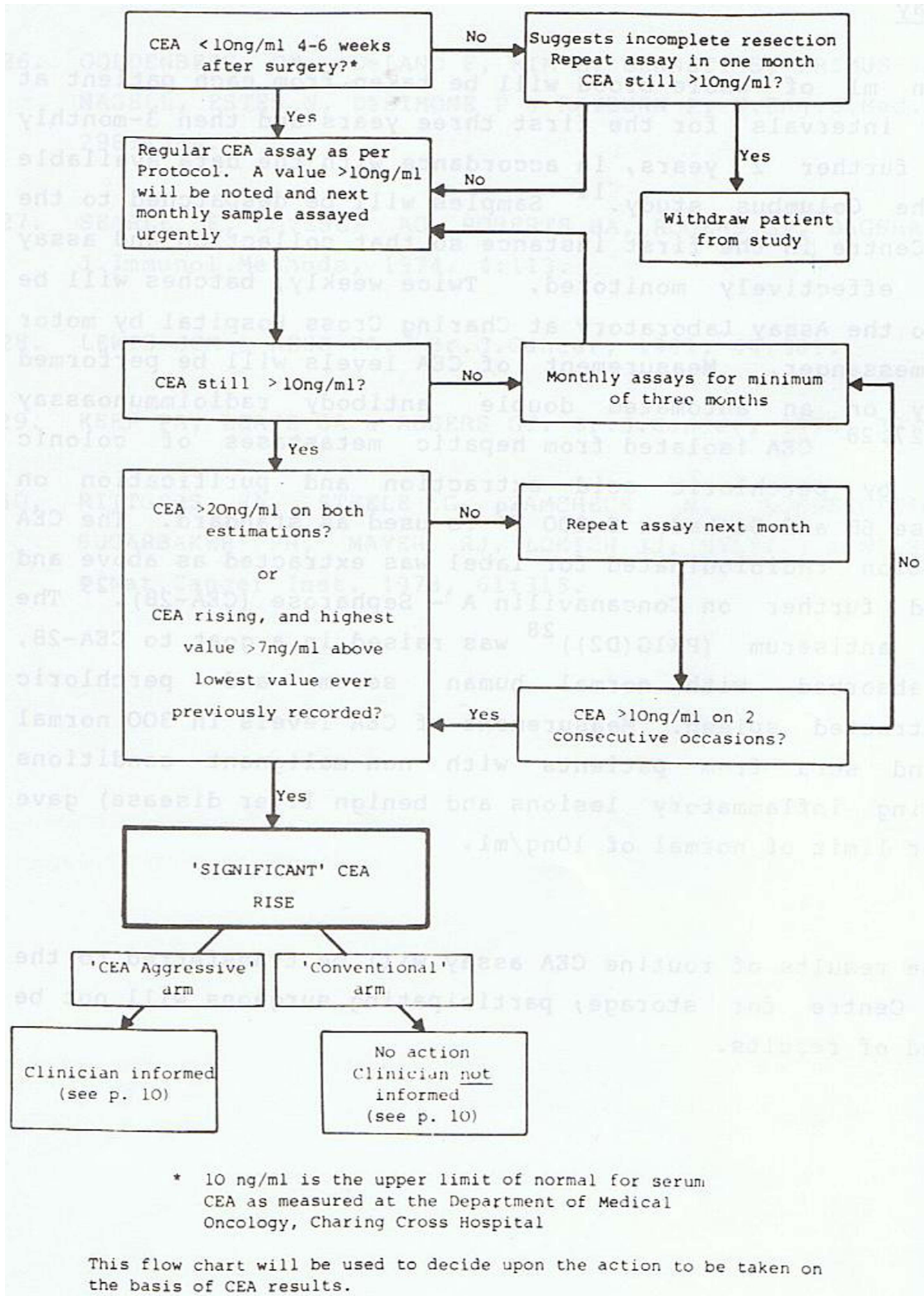

Figure 4 Decision-making algorithm for CEA to trigger second-look surgery. ${ }^{14}$

pelvic cavity, the small bowel, the mesentery, the retroperitoneum, the colon and rectum and the anastomosis was undertaken. The liver was fully mobilised to determine whether any tumour was present. Detailed dissection of the pelvic and retroperitoneal areas and therapeutic resection were then carried out with the objective of total extirpation of all recurrence. Complete data recording of the procedure along with the results of the histology of all potentially involved sites was required by the trial's office.
For patients in whom a radical resection was achieved after second-look surgery (motivated either on clinical information or because the patient had been randomised to the 'Aggressive' arm) the follow-up schedules for clinical examination and blood sampling reverted to those following the primary operation. However, for patients randomised to the 'Aggressive' arm, clinicians were immediately notified of any further CEA levels above $10 \mathrm{ng} / \mathrm{mL}$. 


\section{Death}

Every patient registered onto the study was 'flagged' with the OPCS (now ONS) which provides automatic notification of date of death. This enabled the trial centre to receive certified cause of death for all patients.

\section{Trial oversight}

A Data Monitoring Sub-Committee (DMSC) composed of Working Party members not entering patients into the trial was asked to review the data after the first 100 patients had been randomised, which occurred in January 1988, and again after 200 patients had been randomised in February 1993. At this point it was recommended by the Data Monitoring Committee that the trial be stopped since it was very unlikely that any clinically important advantage would be demonstrated for patients undergoing second-look surgery.

\section{Methods of the RIAT process}

Data

The RIAT restorative authors had been warned by the statisticians called in to look at the data in 2003-2004 that 'the databases were corrupted with key variables no longer abstractable' (McConkey C. Chris McConkey e-mail 1 October 2009, unpublished work and Gray R. Richard Gray e-mail 1 October 2009, unpublished work). We found that the data on paper and on file were accessible and we had no reason to doubt the veracity of individual items. We found that the electronic files had numerous problems with formatting which made the files on the 1447 individual patients difficult to handle but that the data entries were not themselves corrupted.

One of the RIAT restorative authors (KM) had worked in the Trials Centre during the time the CEA trial data were being accrued and knew the systems in use and their changes but was not directly involved in this trial at any stage.

The questions raised and the problems encountered were resolved as follows:

- The codes indicating that a patient had met the criteria for CEA elevation and whether they were randomised to 'Aggressive' or 'Conventional' arm were preserved and tallied with the number in the 1994 manuscript (Northover and Houghton, 1994, unpublished work).

- There were variations in the way dates were recorded in the database. There had been migrations of data from a 'Prime' server using 'Universe' to 'Excel' and the interpretation of the present authors, with information from contemporary witnesses was that in undertaking the task operators did not always correctly specify these data as 'dates' when importing, and/or allowed them to be converted to American date formats. These errors prevented calculations and would have defeated running a survival analysis without correction of the file entries. The dates were however visually readable and not 'corrupt'. Some could be corrected by running current versions of software. Others were manually corrected by re-entering them in a Microsoft date format. Paper records were available to resolve uncertainties.

- The next problem was in linking these three groups of patients (randomised to 'Aggressive', randomised to 'Conventional' and not randomised) to the dates for survival analysis. Individual patients were uniquely identified in the files by seven digit strings to which letters had been added at the beginning and end, possibly for trial administrators' checklists or subgroup identification. Once we had established that the initial and terminal letters were redundant for analysis of the primary endpoint, we were able to write the code to restore the seven digit strings.

- It was evident that the seven digits did not represent a simple sequence but certain positions identified particular characteristics, such as participating centre. We recognised a consistent pattern of mismatch in the fourth digit, a zero in one file was an eight in the other with all other digits remaining the same. It was suggested to us that the fourth digit replacement was used to identify patients suitable for post hoc subgroup analyses but no documentation was found to confirm this. By checking back to the dates of birth we were able to confirm that this systematic correction resolved the problem and most of the data were then usable.

- By ranking all the data in the paired files for line by line visual inspection residual discrepancies were identified. Scrutinising the digit strings allowed for seven of the remaining eight pairs to be reconciled and verified on dates of birth. We failed to resolve only 1 of 1447 records in each file. This patient had not been randomised.

- Inspection of the accrual of death dates was discontinuous for a couple of years suggesting a lapse in either recovery or entry. The current trials centre obtained permission to re-run the ONS search in July 2012.

In summary, we identified several problems but they were systematic and not random (we would not use the value-laden word 'corrupted'). We were able to rectify the formatting errors and verify that the data used for our analysis were correct. The Kaplan-Meier analysis was re-run.

\section{RESULTS}

\section{The original main results 1994}

The study opened to recruitment in November 1982 and was closed by the Working Party, on the acceptance of a recommendation from the DMSC, on 17 February 1993. During this period 1447 patients were registered by 73 participating clinicians in 58 hospitals in the UK. Of these $39(2.7 \%)$ were deemed ineligible since their CEA did not fall below $10 \mathrm{ng} / \mathrm{mL}$ by 6 weeks after surgery. A further 173 patients were excluded from 
analysis; 4 did not have a confirmed diagnosis of adenocarcinoma, 6 were considered unfit for continued monitoring, 4 had a previous and 1 a simultaneous non-colorectal malignancy, 2 had metastatic disease and $156(10.8 \%)$ never complied with the requirement for monthly blood sampling or only did so for 3 months or less (figure 5).

Of 1235 patients who continued in the trial, $80 \%$ achieved a greater than $60 \%$ compliance with blood sampling, while $12.5 \%$ registered between $40 \%$ and $59 \%$ of the required samples and only $7.5 \%$ had compliance of less than $40 \%$ The majority of randomisations (160/ $216 ; 74 \%$ ) were prior to the second anniversary of the primary diagnosis. Three patients randomised had prior recurrent ${ }^{1}$ or metachronous (Slack et al, 1982, unpublished work) disease detected clinically, without a rise in CEA and were operated on.

Two hundred and sixteen patients developed a 'significant' rise in CEA and as no recurrent disease had been recorded at their latest trial follow-up, they were randomised by the Trial Office (108 to each arm). The median time from primary surgery to randomisation was 403 days, (range 103-1754) with no statistical difference between the two groups (Northover and Houghton, 1994, unpublished work).

The characteristics of patients in the two groups are given in table 1.

The stage mix of 980 patients who were eligible for inclusion in the randomised trial but who did not have a CEA rise as defined was Dukes' A 15.1\%, B 55.2\%, C1 $23.3 \%$, C2 $6.4 \%$.

Of the patients randomised to the 'Aggressive' arm 83 (77\%) had recurrent cancer identified and 62 (57\%) patients had 'second-look' surgery. In patients randomised to the 'Conventional' arm 89 (82\%) had developed recurrent disease by the date of analysis. In $26(24 \%)$ of these patients second-look procedures were undertaken. By February 1993, 91/108 in the the 'Aggressive arm' had died and 88/108 patients had died in the 'Conventional' arm (relative risk $=1.16,95 \%$ CI 0.87 to 1.37 ) (Northover and Houghton, 1994, unpublished work). It was considered by the data monitoring committee to be "highly unlikely that any survival advantage would be demonstrated for patients undergoing second-look surgery'. This was communicated to the chief investigator.

\section{RIAT restoration and updated survival analysis}

The data were restored by the RIAT authors for 1446 of 1447 patients to the extent that the RIAT authors were confident of their dates of birth, death and whether they met criteria for entry into the controlled trial and then to which arm they were allocated.

The electronic records were intact with respect to the identity of the patients, which patients had reached the criteria for randomisation, and the trial arm to which they had been randomly allocated for all 216 patients who were randomised. The sex, age, primary site and Dukes' stage as recorded in the 1994 manuscript are shown in table 1 .

Certification of death was obtained from ONS on behalf of the RIAT restorative authors for 204 of 216 randomised patients who died between 17 October 1983 and 8 September 2011. There were equal numbers of patients in the two arms (108) and equal numbers of death dates were retrieved (102). We also have dates of death in 862 of the 1230 patients who were not randomised. Kaplan-Meier analysis in these three groups is shown in figure 6, showing survival of the 1230
Figure 5 Flow chart of enrolled and ultimately randomised patients. 'Blind' in the bottom left box means that the clinical teams were unaware of the elevated carcinoembryonic antigen discovered and were unaware that the patients have been randomised. They were indistinguishable among the 1230 non-randomised patients who were being followed up (see figure 6).

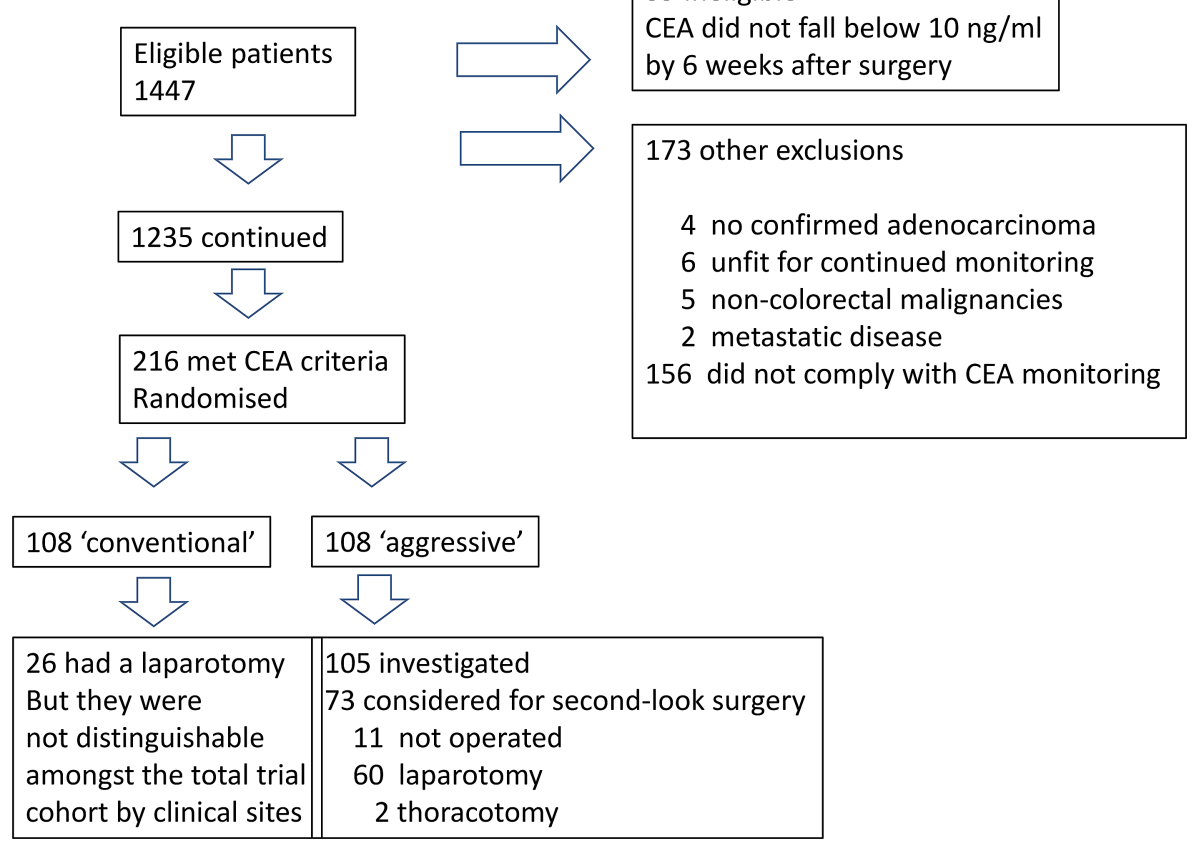


Table 1 Age, sex and colorectal cancer stage of 216 randomised patients

\begin{tabular}{lll}
\hline & $\begin{array}{l}\text { Aggressive } \\
\mathbf{N}=108\end{array}$ & $\begin{array}{l}\text { Conventional } \\
\mathbf{N}=108\end{array}$ \\
\hline Sex male (\%) & $60(56 \%)$ & $68(63 \%)$ \\
Age years, median & $64(33-75)$ & $62(35-75)$ \\
and range & & \\
Pathological stage & $\mathrm{N}(\%)$ & $\mathrm{N}(\%)$ \\
$\quad$ Dukes' A & $5(4.6)$ & $5(4.6)$ \\
Dukes' B & $46(42.6)$ & $49(45.4)$ \\
Dukes' C1 & $36(33.3)$ & $38(35.2)$ \\
Dukes' C2 & $17(15.7)$ & $10(9.3)$ \\
Missing & $4(3.7)$ & $6(5.6)$ \\
\hline
\end{tabular}

participants who entered the trial but were not randomised and the 108 participants randomised into each arm.

The lead time conferred by CEA monitoring, defined as the median time to clinically detected disease for patients randomised to the 'Conventional' arm, was 323 days (95\% CI 203 to 443). This analysis included censored observations on 23 patients, however only 5 of these had a censored time less than the lead time. It was regarded as unlikely, therefore, that the lead time would decrease as further events occur. The analysis presented to the British Oncological Association in 1994 showed that at 3, 6 and 12 months the CEA versus clinical detection rates for recurrence were $88 \%$ vs $18 \%, 95 \%$ vs $44 \%$ and $97 \%$ vs $70 \%$. The RIAT authors did not repeat this analysis.

\section{DISCUSSION}

We have restored data sufficient to achieve the primary outcome of interest as specified by the CEA trialists:

Does a policy of CEA-prompted second-look surgery following 'curative' resection of colorectal cancer produce a decrease in morbidity and mortality due to tumour recurrence, despite sequelae of second look surgery?

The answer is that acting on CEA elevation by secondlook surgery did not reduce mortality compared with patients in whom similar CEA elevation remained unknown. This negative finding led to the closing of the trial in $1994^{123}$ and we confirm it here. There was a small non-significant excess of deaths in the 'Aggressive' arm. The burden of morbidity attributable to the greater number of investigations and operations was not captured by the trial protocol nor indeed the 'needless anxiety' which concerned Moertel et $a l^{22}$ and the authors of the CEA trial protocol (Slack et al, 1982, unpublished work).

The second planned analysis was to obtain an accurate picture of the 'lead time' produced by CEA compared to clinical pick up of patients with recurrence. CEA monitoring did pick up patients considerably sooner

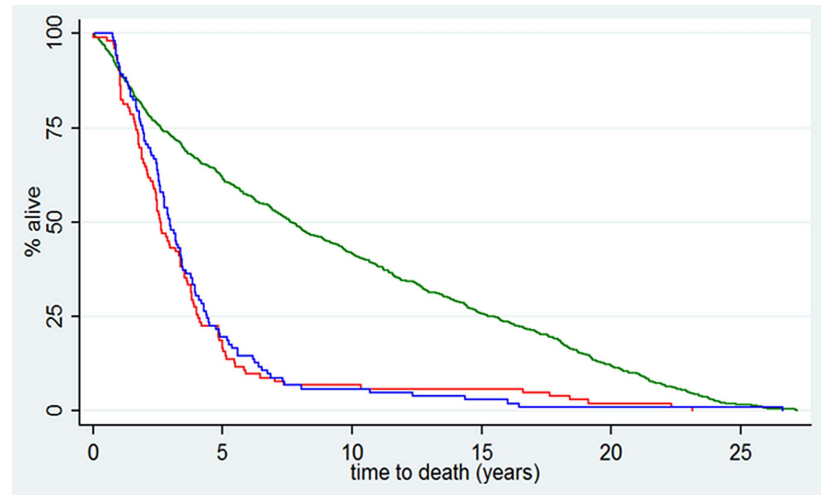

Figure 6 Survival from date of recruitment into the carcinoembryonic antigen (CEA) Second-Look Trial $(\mathrm{N}=1446)$ following potentially curative colorectal cancer surgery. Patients who had CEA elevation according to the trial criteria $(\mathrm{N}=216)$ were randomly allocated in equal groups to have CEA revealed to their surgeons (red) or concealed (blue). Date of death was confirmed from Office for National Statistics in 104/108 in each arm. The green line is for all other patients. Some ( $\mathrm{N}=862$ of 1230 ) would have had clinically evident early recurrence precluding randomisation. The initial plateau is an illustration of a death-free interval ${ }^{44}$ or 'immortal time bias'45 Patients in prospective studies may have a built in obligatory survival time from some starting point in order to attain the requirements to be included in the data set. This is an artefact but may be absorbed into survival time adding to and not readily distinguished from survival time attributed to treatment.

than the clinical methods available at the time by 11 months (95\% CI 7 to 14 months).

CEA monitoring for the purpose of early detection of asymptomatic cancer is currently recommended at least every 6 months in the first 3 years. In addition a minimum of two CT scans are recommended in the first 3 years. ${ }^{34}$ The FACS trial, recently reported, has also shown no survival advantage from CEA monitoring compared with minimum follow-up. ${ }^{35}$ More operations were performed with 'curative intent' for recurrent cancer in those having more intensive monitoring and there were more deaths $(18.2 \%$ (164/901) vs $15.9 \%$ (48/301); difference, $2.3 \%$; $95 \%$ CI $-2.6 \%$ to $7.1 \%$ ). These results are similar to the findings in the CEA trial. Although the phrase 'curative intent' occurs about 40 times in the manuscript, better survival was not achieved with any of the three monitoring schedules compared with minimum follow-up.

The third and fourth intentions set out by the CEA trialists were $(\mathrm{C})$ to obtain further data relating CEA levels to tumour histology and topography and (D) a large database on the natural history of colorectal cancer. Multiple CEA assay results exist in the data we hold for 1446 patients and it would be possible to link these to survival as a result of the RIAT restorative work.

With respect to the natural history of colorectal cancer although we trust the death certification data for the date of death it has been shown that 'at least a third of all death certificates are likely to be incorrect'. ${ }^{36}$ No 
doubt aware of this and seeking much more detailed information, the CEA trialists had asked for detailed postmortem examinations.

It appears that it was disagreement concerning explanatory analyses which contributed to the failure to publish the primary outcome of interest (Northover and Houghton, 1994, unpublished work). The purpose of such analyses would be to discover subsets of patients in whom there was a benefit from the intervention under evaluation and to thus determine the characteristics of patients in whom the intervention might have had a beneficial effect by analysis of mediators and moderators. $^{37}$ There is a general objection to this exercise because it can lead to spurious associations. ${ }^{38} \quad 39$ Furthermore when there is no overall benefit found, as in the CEA Second-Look Trial, any subgroup(s) where there is a positive association between intervention and outcome must be balanced by one or more other groups where there was net harm. The methods section of the 1994 manuscript states 'Subgroup analyses have been performed to address specific issues but these need to be interpreted with appropriate caution' (Northover and Houghton, 1994, unpublished work). In the event no completed subset analyses were in the 1994 paper and the closing notes between the authors are on the matter of a subset analysis. We have not attempted any in restoring the trial.

The answer to the primary research question was clear in 1993 and was the explicit reason for stopping the trial: it was improbable that a benefit from CEA prompted second-look surgery had been missed and in the absence of benefit there was net harm being done to the patients. The forms of second-look surgery now widely practiced in colorectal cancer are liver and lung resection of metastases.

- Full mobilisation of the liver at second-look laparotomy was included in the CEA trial protocol. Hepatic resection has entered routine practice based on observational data ${ }^{40}$ and an opportunity to do a randomised trial, for which a power calculation was proposed in 1992 from the Mayo Clinic, ${ }^{41}$ was not taken. ${ }^{24}$

- Two patients had a thoracotomy prompted by CEA elevation. Pulmonary metastasectomy for colorectal cancer is, after primary lung cancer, the second commonest thoracic cancer operation and is the subject of an ongoing randomised controlled trial. ${ }^{42}$

The CEA trial findings have been corroborated by the larger FACS trial. If the CEA trial results had been made available in 1994, and there is no evident reason why they should not have been, a more critical scrutiny of the evidence base that was used to bring liver and lung metastasectomy into practice ${ }^{24}$ might have been undertaken. The CEA trial was a well-conceived and meticulously executed randomised trial and we hope that publishing it now more than 20 years after its completion will indicate the possibility of more randomised trials in surgery. ${ }^{43}$
Author affiliations

${ }^{1}$ Clinical Operational Research Unit, University College London, London, UK

${ }^{2}$ Sussex Health Outcomes Research \& Education in Cancer (SHORE-C), Brighton \& Sussex Medical School, University of Sussex, Falmer, Brighton, UK

${ }^{3}$ Department of Cardiothoracic Surgery, NHLI, Imperial College London, London, UK

${ }^{4}$ Department of Surgery, University College London, London, UK

Acknowledgements The RIAT authors are grateful to Jonathan Ledermann, Director of the Cancer Research UK \& UCL Cancer Trials Centre, University College London, (where the CEA files were stored) and Sharon Forsyth for her assistance in accessing the CEA trial data and updating the Office for National Statistics records for death registration. The authors also acknowledge the assistance of Sonya Crowe of UCL's Clinical Operational Research Unit in technical aspects of restoring the electronic files to a usable state. The RIAT authors met with the following persons who were members of the 1982 Working Party at the Cancer Research Campaign Clinical Trials Centre (CRC CTC) King's College Hospital Medical School, London and/or were listed as contributors in the 1994 manuscript from the CRC CTC at the Rayne Institute, 123 Coldharbour Lane, London SE5 9NU: M Baum, RHJ Begent, H Ellis, J Houghton, M Irving, CA Lennon, JMA Northover, WW Slack and CB Wood. They are particularly grateful to JMAN who met with TT and RCGR at the Royal Society of Medicine in London on Monday 10 February 2014 and read and commented on a near final version of the manuscript and agreed our interpretation of the trial results. The principal findings reported here corroborate those in his letter to JAMA in 1994. JMAN helped us understand the sequence of events leading to the closure of the trial and subsequent lapse in writing up the full report.

Contributors TT instigated the recovery of the data, worked on the database recovery described in the manuscript and wrote the first and edited the final version of the manuscript. KM navigated the data files and worked on the database recovery described in the manuscript. FF performed the analysis of the recovered data and the presentation of the analysis. CR negotiated access to the data and with TT contacted and interviewed the members of the original trial team. All authors have read and contributed to successive iterations of the manuscript and approve the submitted version.

Funding The CEA Second-Look Trial opened in 1982 and was jointly funded by Cancer Research Campaign and the National Institute of Health. The restoration of the trial was unfunded. The four RIAT restorative authors gave their time unpaid.

Competing interests FF is partly funded by the British Heart Foundation.

Ethics approval Kings College Hospital 1982.

Provenance and peer review Not commissioned; externally peer reviewed.

Data sharing statement The authors are prepared to share the anonymised electronic data in their possession. The chief investigator (JMAN) and the chair of data monitoring committee (MB) provided a signed agreement on 21 February 2014 to allow access to the archived paper records and electronic files (held by UCLCTC) at the discretion of the Restoring Invisible and Abandoned Trials (RIAT) authors. Survival data for two randomised and one non-randomised groups can be accessed in the Dryad data repository: doi:10.5061/dryad.k8t8b.

Open Access This is an Open Access article distributed in accordance with the Creative Commons Attribution Non Commercial (CC BY-NC 3.0) license, which permits others to distribute, remix, adapt, build upon this work noncommercially, and license their derivative works on different terms, provided the original work is properly cited and the use is non-commercial. See: http:// creativecommons.org/licenses/by-nc/3.0/

\section{REFERENCES}

1. Northover J, Houghton J, Lennon T. CEA to detect recurrence of colon cancer [Letter]. JAMA 1994;272:31.

2. Doshi $P$, Dickersin K, Healy D, et al. Restoring invisible and abandoned trials: a call for people to publish the findings. BMJ 2013;346:f2865 
3. Loder E, Godlee F, Barbour V, et al. Restoring the integrity of the clinical trial evidence base. BMJ 2013;346:f3601.

4. Gill PG, Morris PJ. The survival of patients with colorectal cancer treated in a regional hospital. Br J Surg 1978;65:17-20.

5. Slaney G. Results of treatment of carcinoma of the colon and rectum. In: Irvine T, ed. Modern Trends in Surgery 3rd ed. London: Butterworths Group; 1971:69-89.

6. Welch JP, Donaldson GA. Detection and treatment of recurrent cancer of the colon and rectum. Am J Surg 1978;135:505-11.

7. Goligher JC. Surgery of the anus, colon and rectum. 4th ed. London: Bailliere Tindall, 1980.

8. Martin EW Jr, Cooperman M, King G, et al. A retrospective and prospective study of serial CEA determinations in the early detection of recurrent colon cancer. Am J Surg 1979;137:167-9.

9. Cochrane JP, Williams JT, Faber RG, et al. Value of outpatient follow-up after curative surgery for carcinoma of the large bowel. BMJ 1980;280:593-5.

10. Ekman CA, Gustavson J, Henning A. Value of a follow-up study of recurrent carcinoma of the colon and rectum. Surg Gynecol Obstet 1977; 145:895-7.

11. Bacon HE, Berkley JL. The rationale of re-resection for recurrent cancer of the colon and rectum. Dis Colon Rectum 1959;2:549-54.

12. Polk HC Jr, Spratt JS Jr. Recurrent colorectal carcinoma: detection, treatment, and other considerations. Surgery 1971;69:9-23.

13. Ellis $\mathrm{H}$. Is a 'second look operation' justified in suspected recurrences after abdominal cancer surgery? Br J Surg 1975;62:830-2.

14. Wangensteen $\mathrm{O}$, Lewis $\mathrm{F}$, Arhelger $\mathrm{S}$, et al. An interim report upon the second look procedure for cancer of the stomach, colon, and rectum and for limited intraperitoneal carcinosis. Surg Gynecol Obstet 1954;99:257-67.

15. Martin EW Jr, Cooperman M, Carey LC, et al. Sixty second-look procedures indicated primarily by rise in serial carcinoembryonic antigen. J Surg Res 1980;28:389-94.

16. Staab HJ, Anderer FA, Stumpf E, et al. Carcinoembryonic antigen follow-up and selection of patients for second-look operation in management of gastrointestinal carcinoma. J Surg Oncol 1978;10:273-82.

17. Nicholson JR, Aust JC. Rising carcinoembryonic antigen titers in colorectal carcinoma: an indication for the second-look procedure. Dis Colon Rectum 1978:21:163-4.

18. Mackay AM, Patel S, Carter S, et al. Role of serial plasma C.E.A. assays in detection of recurrent and metastatic colorectal carcinomas. BMJ 1974:4:382-5.

19. Mach JP, Jaeger P, Bertholet MM, et al. Detection of recurrence of large-bowel carcinoma by radioimmunoassay of circulating carcinoembryonic antigen (C.E.A.). Lancet 1974;304:535-40.

20. Goldenberg DM (Chair). Carcinoembryonic antigen: its role as a marker in the management of cancer. Summary of an $\mathrm{NIH}$ consensus statement. BMJ (Clin Res Ed) 1981;282:373-5.

21. Meeker WR Jr. The use and abuse of CEA test in clinical practice. Cancer 1978;41:854-62.

22. Moertel CG, Schutt AJ, Go VL. Carcinoembryonic antigen test for recurrent colorectal carcinoma. Inadequacy for early detection. JAMA 1978;239:1065-6.

23. Lennon T, Houghton J, Northover J. Post operative CEA monitoring and second-look surgery in colorectal cancer: trial results [Abstract]. Br J Cancer 1994;70(Suppl xxii):16.

24. Grunhagen D, Jones RP, Treasure T, et al. The history of adoption of hepatic resection for metastatic colorectal cancer: 1984-95. Crit Rev Oncol Hematol 2013;86:222-31.

25. Embun R, Fiorentino F, Treasure T, et al. Pulmonary metastasectomy in colorectal cancer: a prospective study of demography and clinical characteristics of 543 patients in the
Spanish colorectal metastasectomy registry (GECMP-CCR). BMJ Open 2013;3:pii: e002787.

26. Treasure T, Utley M, Hunt I. When professional opinion is not enough: surgical resection of pulmonary metastases. BMJ 2007;334:831-2.

27. Fiorentino F, Treasure T. Pulmonary metastasectomy for colorectal cancer: making the case for a randomized controlled trial in the zone of uncertainty. J Thorac Cardiovasc Surg 2013;146:748-52.

28. Fiorentino $\mathrm{F}$, Hunt I, Teoh $\mathrm{K}$, et al. Pulmonary metastasectomy in colorectal cancer: a systematic review and quantitative synthesis. J R Soc Med 2010;103:60-6.

29. Fiorentino F, Vasilakis C, Treasure T. Clinical reports of pulmonary metastasectomy for colorectal cancer: a citation network analysis. Br J Cancer 2011;104:1085-97.

30. Treasure T. Carcinoembryonic antigen: its place in decision making for pulmonary metastasectomy in colorectal cancer. J Thorac Oncol 2010;5(6 Suppl 2):S179-81.

31. Searle F, Lovesey AC, Roberts BA, et al. Radioimmunoassay methods for carcinoembryonic antigen. $J$ Immunol Methods 1974;4:113-25.

32. Lewis JC, Keep PA. Relationship of serum CEA levels to tumour size and CEA content in nude mice bearing colonic-tumour xenografts. Br J Cancer 1981;44:381-7.

33. Keep PA, Leake BA, Rogers GT. Extraction of CEA from tumour tissue, foetal colon and patients' sera, and the effect of perchloric acid. Br J Cancer 1978;37:171-82.

34. Poston G, Guideline Development Group. Colorectal cancer: the diagnosis and management of colorectal cancer. National Institute for Health and Clinical Excellence, 2011 [cited \& Oct 2012]. http:// guidance.nice.org.uk/CG131/Guidance/pdf/English

35. Primrose JN, Perera R, Gray A, et al. Effect of 3 to 5 years of scheduled CEA and CT follow-up to detect recurrence of colorectal cancer: the FACS randomized clinical trial. JAMA 2014:311:263-70.

36. NCEPOD (National Confidential Enquiry into Patient Outcome and Death. The Coroner's autopsy: do we deserve better? 2006. National Confidential Enquiry into Patient Outcome and Death

37. Kraemer HC. Toward non-parametric and clinically meaningful moderators and mediators. Stat Med 2008;27:1679-92.

38. Randomised trial of intravenous streptokinase, oral aspirin, both, or neither among 17,187 cases of suspected acute myocardial infarction: ISIS-2. ISIS-2 (Second International Study of Infarct Survival) Collaborative Group. Lancet 1988;2:349-60.

39. Horton R. From star signs to trial guidelines. Lancet 2000;355:1033-4.

40. Morris EJ, Forman D, Thomas JD, et al. Surgical management and outcomes of colorectal cancer liver metastases. Br J Surg 2010;97:1110-18.

41. Rosen CB, Nagorney DM, Taswell HF, et al. Perioperative blood transfusion and determinants of survival after liver resection for metastatic colorectal carcinoma. Ann Surg 1992;216:493-504.

42. Treasure T, Leonard P. Pulmonary metastasectomy in colorectal cancer. Br J Surg 2013;100:1403-4.

43. Treasure T, Morton D. GRIST: Growing Recruitment in Interventional and Surgical Trials. J R Soc Med 2012;105:140-1.

44. Utley M, Treasure T, Linklater K, et al. Better out than in? The resection of pulmonary metastases from colorectal tumours. In: Xie X, Lorca F, Marcon E. eds. Operations research for health care engineering: proceedings of the 33rd international conference on operational research applied to health services. Saint-Etienne: Publications de l'Universitaire de Saint-Etienne, 2008:493-500.

45. Levesque LE, Hanley JA, Kezouh A, et al. Problem of immortal time bias in cohort studies: example using statins for preventing progression of diabetes. BMJ 2010;340:b5087. 